\title{
In Vitro Gut Modeling as a Tool for Adaptive Evolutionary Engineering of Lactiplantibacillus plantarum
}

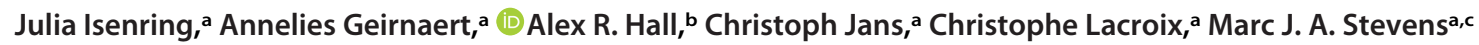 \\ aLaboratory of Food Biotechnology, Institute of Food, Nutrition and Health, ETH Zurich, Zürich, Switzerland \\ bInstitute of Integrative Biology, ETH Zurich, Zürich, Switzerland \\ Institute for Food Hygiene and Safety, University of Zurich, Zürich, Switzerland
}

ABSTRACT Research and marketing of probiotics demand holistic strain improvement considering both the biotic and abiotic gut environment. Here, we aim to establish the continuous in vitro colonic fermentation model PolyFerms as a tool for adaptive evolutionary engineering. Immobilized fecal microbiota from adult donors were steadily cultivated up to 72 days in PolyFermS reactors, providing a long-term compositional and functional stable ecosystem akin to the donor's gut. Inoculation of the gut microbiota with immobilized or planktonic Lactiplantibacillus plantarum NZ3400, a derivative of the probiotic model strain WCFS1, led to successful colonization. Whole-genome sequencing of 45 recovered strains revealed mutations in 16 genes involved in signaling, metabolism, transport, and cell surface. Remarkably, mutations in LP_RS14990, LP_RS15205, and intergenic region LP_RS05100<LP_RS05095 were found in recovered strains from different adaptation experiments. Combined addition of the reference strain NZ3400 and each of those mutants to the gut microbiota resulted in increased abundance of the corresponding mutant in PolyFermS microbiota after 10 days, showing the beneficial nature of these mutations. Our data show that the PolyFermS system is a suitable technology to generate adapted mutants for colonization under colonic conditions. Analysis thereof will provide knowledge about factors involved in gut microbiota colonization and persistence.

IMPORTANCE Improvement of bacterial strains in regard to specific abiotic environmental factors is broadly used to enhance strain characteristics for processing and product quality. However, there is currently no multidimensional probiotic strain improvement approach for both abiotic and biotic factors of a colon microbiota. The continuous PolyFerms fermentation model allows stable and reproducible continuous cultivation of colonic microbiota and provides conditions akin to the host gut with high control and easy sampling. This study investigated the suitability of PolyFermS for adaptive evolutionary engineering of a probiotic model organism for lactobacilli, Lactiplantibacillus plantarum, to an adult human colonic microbiota. The application of PolyFermS controlled gut microbiota environment led to adaptive evolution of $L$. plantarum strains for enhanced gut colonization characteristics. This novel tool for strain improvement can be used to reveal relevant factors involved in gut microbiota colonization and develop adapted probiotic strains with improved functionality in the gut.

KEYWORDS adaptive evolutionary engineering, colonic microbiota, in vitro gut modeling, Lactiplantibacillus plantarum

icroorganisms play a pivotal role in pharmaceutical, biotechnological, and food industries. The last depends heavily on microorganisms for starter cultures, biopreservation agents, and flavor producers (1-3). Moreover, since the 1990s, there has been an increase in the production of probiotics, which are "live microorganisms that, when administered in adequate amounts, confer a health benefit on the host" (4).
Citation Isenring J, Geirnaert A, Hall AR, Jans C, Lacroix C, Stevens MJA. 2021. In vitro gut modeling as a tool for adaptive evolutionary engineering of Lactiplantibacillus plantarum. mSystems 6:e01085-20. https://doi.org/10 $.1128 / \mathrm{mSystems} .01085-20$. Editor Daniel Garrido, Pontificia Universidad Catolica de Chile

Copyright $\odot 2021$ Isenring et al. This is an open-access article distributed under the terms of the Creative Commons Attribution 4.0 International license.

Address correspondence to Christophe Lacroix, christophe.lacroix@hest.ethz.ch.

Received 21 October 2020

Accepted 26 February 2021

Published 13 April 2021 
Strain improvement of probiotic bacteria is of major importance to meet consumer demands for functional foods and enhance competitiveness of probiotic strains. However, it demands a multidimensional approach since biotic and abiotic factors are involved.

A promising solution for strain improvement is evolutionary engineering, which steers microbial evolution by exerting selective pressure (5-7). Desired mutants can be selected based on, e.g., growth rate, increased survival, or retention time. This method is feasible with bacteria because short generation times and large population sizes facilitate rapid emergence and selective sweeps of mutants (8-11). It is a well-established approach to improve targeted strain characteristics like the acidification rate of Lactococcus lactis (12), growth of Escherichia coli (13), or enhanced succinate production in Actinobacillus and Mannheimia $(10,14,15)$. Nonetheless, the potential of evolutionary engineering as multidimensional engineering within microbial consortia is not well established yet. Previously, the residence time of Lactiplantibacillus plantarum in the murine digestive tract was increased after repetitive administration of the longestpersisting L. plantarum (16). However, in vivo models like mice have societal, ethical, and monetary restrictions and might therefore be replaced by in vitro models. Furthermore, gastrointestinal physiology and gut species composition of mice are different from humans, possibly limiting the translation $(17,18)$.

Continuous fermentation models are best suited for in vitro cultivation of gut microbiota in conditions akin to the gut $(19,20)$. Different PolyFermS models were successfully developed for cultivating colonic microbiota of humans of different ages and conditions and swine, murine, and chicken cecum microbiota (21-25). The continuous PolyFerms model allows testing several treatments in parallel in second-stage treatment reactors (TRs) seeded with the same gut microbiota produced in the inoculum reactor (IR) containing immobilized microbiota (21). Gut microbiota immobilization in polysaccharide gel beads leads to the maintenance of high cell density, long-term stability due to prevention of cell washout, and diversity of the simulated gut microbiota $(23,26,27)$. It moreover creates a sessile bacterial fraction on the gel beads and a planktonic fraction resulting from the growth and release of sessile bacteria and further growth of planktonic cells in the bulk medium $(27,28)$. This mimics the gastrointestinal environment consisting of free and biofilm- or mucus-associated bacteria (29, 30). The PolyFerms colonic fermentation model enables operation up to several months in a highly controllable environment with multiple parameters to operate on, which is needed for evolutionary adaptation (26). We therefore hypothesized that the PolyFerms model can provide a long-term stable gut microbiota akin to the human adult colon that allows for adaptive evolution of an exogenous single strain.

In this study, we investigated the PolyFerms fermentation model as a novel tool for strain improvement via adaptive evolutionary engineering, using $L$. plantarum as a model strain. $L$. plantarum originates from fermented foods $(31,32)$ and is detected at low levels in approximately half of healthy human gut microbiota (33). L. plantarum WCFS1 is a well-characterized model strain for transient probiotic lactobacilli $(34,35)$. A WCFS1 derivative harboring a chloramphenicol (CM) resistance gene for tracking was cultivated in PolyFerms reactors inoculated with immobilized adult fecal microbiota for at least 100 generations. Engineered strains were phenotypically and genotypically characterized and tested for improved colonization in the PolyFermS model.

\section{RESULTS}

The PolyFerms model allows stable cultivation of adult gut microbiota. The PolyFerms model operated to mimic the adult proximal colon was used to provide a gut microbiota environment for evolutionary adaptation of L. plantarum NZ3400. Adaptation of immobilized L. plantarum was performed in IR1, and adaptation of planktonic L. plantarum was tested in TRs continuously inoculated with IR2 microbiota (Fig. 1). Metabolic stability of IR1 (see Fig. S2A in the supplemental material) was achieved after 1 week with main short-chain fatty acids (SCFAs) acetate, propionate, and butyrate at $73 \pm 7,21 \pm 4$, and $19 \pm 3 \mathrm{mM}$ over a 2-month fermentation, respectively. IR1 microbiota 
A

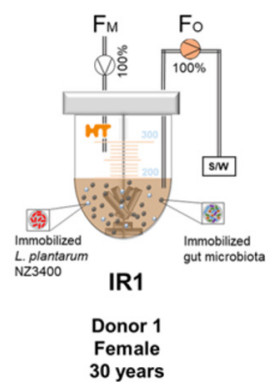

B

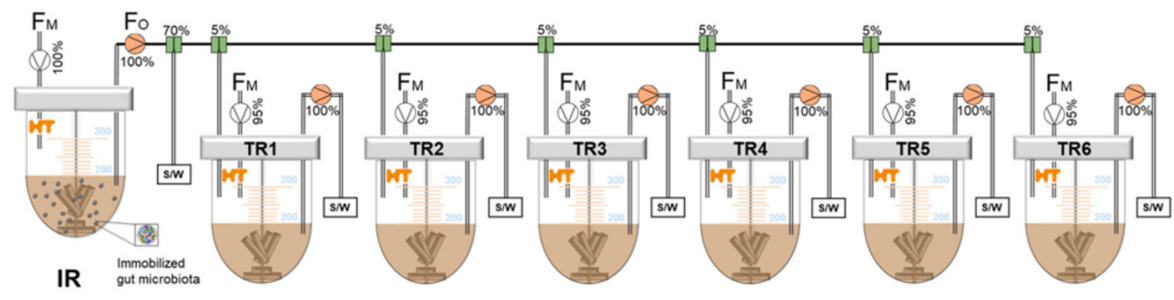

\begin{tabular}{|c|c|c|c|c|c|c|c|c|}
\hline & & & TR1 & TR2 & TR3 & TR4 & TR5 & TR6 \\
\hline \multirow{8}{*}{$\begin{array}{c}\text { Donor } 2 \\
\text { Male } \\
27 \text { years }\end{array}$} & \multirow{4}{*}{$\begin{array}{l}\text { Treatment } \\
\text { period } 1\end{array}$} & Strain & NZ3400B & NZ3400B & IA10 & IA01/NZ3400B & & \\
\hline & & $\begin{array}{l}\text { Supplemented at } \\
\text { (CFU/ml) }\end{array}$ & $1.00 E+06$ & $1.00 \mathrm{E}+09$ & $1.00 \mathrm{E}+09$ & $1.00 E+09$ & & \\
\hline & & $\begin{array}{l}\text { Cultivation for } \\
\text { (days) }\end{array}$ & 44 & 72 & 23 & 10 & & \\
\hline & & Purpose & $\begin{array}{l}\text { Planktonic } \\
\text { adaptation }\end{array}$ & $\begin{array}{l}\text { Long-term planktonic } \\
\text { adaptation }\end{array}$ & $\begin{array}{l}\text { Evaluation } \\
\text { mutated strain }\end{array}$ & Competition & & \\
\hline & \multirow{4}{*}{$\begin{array}{l}\text { Treatment } \\
\text { period } 2\end{array}$} & Strain & NZ3400B & & IA10 & IA01/NZ3400B & & \\
\hline & & $\begin{array}{l}\text { Supplemented at } \\
\text { (CFU/ml) }\end{array}$ & $1.00 \mathrm{E}+09$ & & $1.00 \mathrm{E}+09$ & $1.00 \mathrm{E}+09$ & & \\
\hline & & $\begin{array}{l}\text { Cultivation for } \\
\text { (days) }\end{array}$ & 23 & & 23 & 10 & & \\
\hline & & Purpose & $\begin{array}{l}\text { Planktonic } \\
\text { adaptation }\end{array}$ & & $\begin{array}{l}\text { Evaluation } \\
\text { mutated strain }\end{array}$ & Competition & & \\
\hline \multirow{4}{*}{$\begin{array}{l}\text { Donor } 3 \\
\text { Female } \\
27 \text { years }\end{array}$} & & Strain & $\begin{array}{c}\text { IA01/ } \\
\text { NZ3400B }\end{array}$ & $\begin{array}{l}\text { IA01/ } \\
\text { NZ3400B }\end{array}$ & $\begin{array}{l}\text { PA1.2_01/ } \\
\text { NZ3400B }\end{array}$ & $\begin{array}{l}\text { PA1.2_01/ } \\
\text { NZ3400B }\end{array}$ & $\begin{array}{l}\text { PA2_06/ } \\
\text { NZ3400B }\end{array}$ & $\begin{array}{l}\text { PA2_06/ } \\
\text { NZ3400B }\end{array}$ \\
\hline & & $\begin{array}{l}\text { Supplemented at } \\
\text { (CFU/ml) }\end{array}$ & $1.00 E+09$ & $1.00 \mathrm{E}+09$ & $1.00 \mathrm{E}+09$ & $1.00 E+09$ & $1.00 \mathrm{E}+09$ & $1.00 E+09$ \\
\hline & & $\begin{array}{l}\text { Cultivation for } \\
\text { (days) }\end{array}$ & 10 & 10 & 10 & 10 & 10 & 10 \\
\hline & & Purpose & Competition & Competition & Competition & Competition & Competition & Competition \\
\hline \multirow{4}{*}{$\begin{array}{c}\text { Donor } 4 \\
\text { Female } \\
31 \text { years }\end{array}$} & & Strain & NZ3400B & NZ3400B & $\Delta L P_{-} R S 14990$ & $\Delta L P_{-} R S 14990$ & $\begin{array}{l}\text { PA2_06/ } \\
\text { NZ3400B }\end{array}$ & $\begin{array}{l}\text { PA2_06/ } \\
\text { NZ3400B }\end{array}$ \\
\hline & & $\begin{array}{l}\text { Supplemented at } \\
\text { (CFU/ml) }\end{array}$ & $1.00 \mathrm{E}+09$ & $1.00 \mathrm{E}+09$ & $1.00 \mathrm{E}+09$ & $1.00 E+09$ & $1.00 \mathrm{E}+09$ & $1.00 E+09$ \\
\hline & & $\begin{array}{l}\text { Cultivation for } \\
\text { (days) }\end{array}$ & 10 & 10 & 10 & 10 & 10 & 10 \\
\hline & & Purpose & $\begin{array}{l}\text { Evaluation } \\
\text { knockout strain }\end{array}$ & $\begin{array}{l}\text { Evaluation knockout } \\
\text { strain }\end{array}$ & $\begin{array}{l}\text { Evaluation } \\
\text { knockout strain }\end{array}$ & $\begin{array}{l}\text { Evaluation knockout } \\
\text { strain }\end{array}$ & Competition & Competition \\
\hline \multirow{4}{*}{ Donor 3} & & Strain & NZ3400B & NZ3400B & $\Delta L P \_R S 14990$ & $\Delta L P_{2} R S 14990$ & & \\
\hline & & $\begin{array}{l}\text { Supplemented at } \\
\text { (CFU/ml) }\end{array}$ & $1.00 E+09$ & $1.00 E+09$ & $1.00 E+09$ & $1.00 E+09$ & & \\
\hline & & $\begin{array}{l}\text { Cultivation for } \\
\text { (days) }\end{array}$ & 9 & 9 & 9 & 9 & & \\
\hline & & Purpose & $\begin{array}{c}\text { Evaluation } \\
\text { knockout strain }\end{array}$ & $\begin{array}{l}\text { Evaluation knockout } \\
\text { strain }\end{array}$ & $\begin{array}{c}\text { Evaluation } \\
\text { knockout strain }\end{array}$ & $\begin{array}{c}\text { Evaluation knockout } \\
\text { strain }\end{array}$ & & \\
\hline
\end{tabular}

FIG 1 PolyFermS setup of immobilized and planktonic adaptation and further experiments in in vitro human adult gut microbiota. (A) Immobilized L. plantarum NZ3400 was added to immobilized fecal gut microbiota in IR1 from donor 1 during a single-stage fermentation. (B) Immobilized fecal microbiota of donors 2 to 4 were cultivated in the inoculum reactor (IR), which was used to inoculate second-stage reactors (TRs) that were supplemented with $L$. plantarum after stabilization. NZ3400, reference strain; NZ3400B, new stock from single-colony isolate of NZ3400; IA10, IA01, PA1.2_01, and PA2_06, recovered L. plantarum mutants; L. plantarum $\triangle L P$ _RS14990, LP_RS14990 gene deletion strain; $F_{M^{\prime}}$ inflow MacFarlane medium; $F_{0}$, reactor outflow; $S / W_{\text {, }}$ sampling/waste.

was dominated by Firmicutes and Bacteroidetes accounting for $44 \% \pm 5 \%$ and $47 \% \pm 2 \%$ of the total population during days 44 to 46 , respectively; $48 \% \pm 7 \%$ and $41 \% \pm 2 \%$ during days 57 to 59 , respectively; and $52 \% \pm 4 \%$ and $36 \% \pm 4 \%$ during days 61 to 65 , respectively (see Fig. S3A in the supplemental material). This stability was also observed on a family level (see Fig. S3B in the supplemental material). IR2 microbiota reached metabolic stability (see Fig. S2B in the supplemental material) after 1 week with the main SCFAs acetate, propionate, and butyrate at $84 \pm 5,32 \pm 8$, and $23 \pm 4 \mathrm{mM}$, respectively, during 2 months of culture. IR2 had a different microbiota composition from IR1 (see Fig. S3C and D in the supplemental material). Bacteroidetes dominated the gut microbiota compared to Firmicutes with $63 \% \pm 1 \%$ and $36 \% \pm 1 \%$ during days 24 to 26 , respectively; $68 \% \pm 4 \%$ and $30 \% \pm 4 \%$ during days 41 to 43 , respectively; and $66 \% \pm 1 \%$ and $30 \% \pm 1 \%$ during days 89 to 91 , respectively (see Fig. S3C in the supplemental material). Stability was maintained up to 90 days on a family level (see Fig. S3D in the supplemental material). Microbiota composition of the IR was successfully transferred to and maintained in the TRs (see Fig. S3D in the supplemental material). Both metabolic activity (see Fig. S2C to E in the supplemental material) and composition were reproduced in the TRs. 
A

\section{Colonization of immobilized \\ L. plantarum NZ3400}

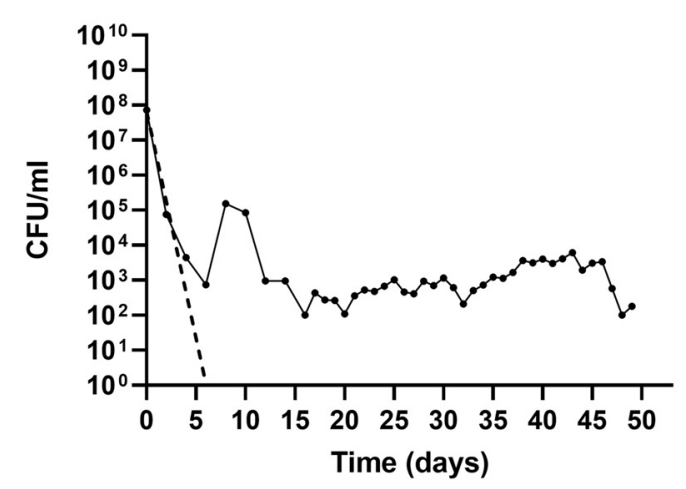

B Colonization of planktonic
L. plantarum NZ3400B

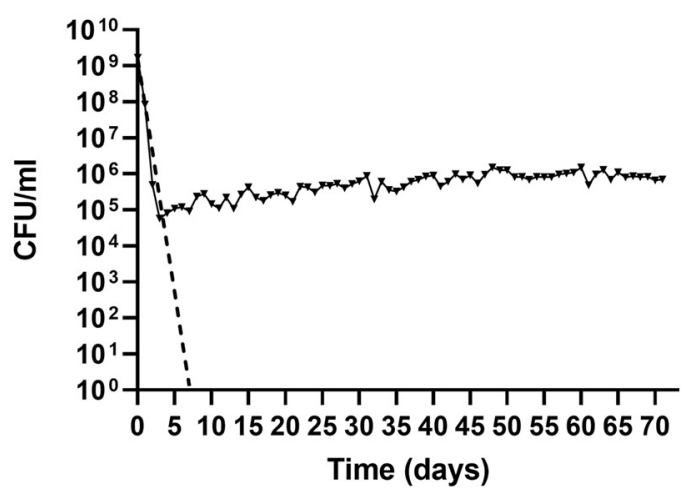

FIG 2 Colonization levels of L. plantarum during immobilized and planktonic adaptation in adult gut microbiota. (A) Immobilized L. plantarum NZ3400 (4 g of colonized beads containing $5 \times 10^{9} \mathrm{CFU}$ L. plantarum/g) was added to the gut microbiota (O) and (B) NZ3400B at $10^{9} \mathrm{CFU} / \mathrm{ml}$ effluent in planktonic state ( $\left.\mathbf{\nabla}\right)$. Dashed line indicates the theoretical washout of the system. Cell count is depicted on the $y$ axis, and days of cultivation in the microbiota are shown on the $x$ axis, where 0 corresponds to the day of $L$. plantarum supplementation.

Prolonged cultivation of $L$. plantarum in in vitro human gut microbiota. To investigate the potential of the continuous in vitro gut fermentation model PolyFermS for evolutionary engineering, immobilized L. plantarum NZ3400 was added to the stabilized microbiota in IR1 at an initial concentration of $10^{8} \mathrm{CFU} / \mathrm{ml}$ effluent. NZ3400 decreased at the rate of the theoretical washout during the first 4 days (Fig. 2A), followed by colonization between $10^{2} \mathrm{CFU} / \mathrm{ml}$ and $10^{4} \mathrm{CFU} / \mathrm{ml}$ during the 50-day fermentation. L. plantarum was able to maintain a self-sustaining population, an observation which will be referred to as colonization.

The PolyFermS model was further evaluated for long-term adaptation of planktonic L. plantarum NZ3400B in TR2, fed by donor 2 gut microbiota. Cell counts decreased from $10^{9}$ to $10^{5} \mathrm{CFU} / \mathrm{ml}$ during the first 4 days after spiking (Fig. $2 \mathrm{~B}$ ), at the rate of the washout. Thereafter, colonization steadily increased to $10^{6} \mathrm{CFU} / \mathrm{ml}$ during 72 days. Repeated supplementation of L. plantarum with $10^{6}$ and $10^{9} \mathrm{CFU} / \mathrm{ml}$ in TR1 (period 1) and TR1 (period 2) resulted in stable colonization at different levels of $1 \times 10^{6}$ and $3 \times$ $10^{4} \mathrm{CFU} / \mathrm{ml}$, respectively. Therefore, L. plantarum colonization at a donor-specific level was demonstrated for more than 50 days and 150 generations.

Recovered $L$. plantarum strains are phenotypically adapted to the gut microbiota environment. To test for $L$. plantarum adaptation during gut microbiota cultivation, recovered strains were grown in SCFA concentrations comparable to those in the gut fermenter. Average growth of strains from immobilized adaptation measured by optical density (OD) was impaired in De Man, Rogosa, and Sharpe (MRS) and MRS supplemented with $30 \mathrm{mM}$ propionate or butyrate compared to the reference strain NZ3400B (Table 1). Strains from the early stage of long-term planktonic adaptation behaved similarly to the reference NZ3400B in all tested media. However, strains from late planktonic adaptation and biofilm grew better in MRS plus acetate $(+0.11$ and +0.07 , respectively), less in standard MRS $(-0.14$ and -0.11 , respectively), and similarly in MRS plus propionate or butyrate (Table 1) compared to NZ3400B. Moreover, strains from biofilm and late planktonic adaptation grew significantly better in the reactoreffluent-mimicking effluent-MacFarlane-sugar (EMS) medium than strains from early adaptation (Table 1).

When clustered according to growth performance in MRS and MRS supplemented with acetate $(50 \mathrm{mM})$, butyrate $(30 \mathrm{mM})$, and propionate $(30 \mathrm{mM})$, strains from immobilized and planktonic adaptation were clearly separated (Fig. 3). Further, strains recovered from immobilized adaptation exhibited a higher growth variability than strains isolated from the long-term planktonic adaptation (see Fig. S4 in the supplemental 
TABLE 1 Growth of recovered L. plantarum strains in different media ${ }^{a}$

\begin{tabular}{|c|c|c|c|c|c|}
\hline L. plantarum origin & MRS & $\begin{array}{l}\text { MRS + acetate } \\
(50 \mathrm{mM})\end{array}$ & $\begin{array}{l}\text { MRS + propionate } \\
(30 \mathrm{mM})\end{array}$ & $\begin{array}{l}\text { MRS + butyrate } \\
(30 \mathrm{mM})\end{array}$ & EMS \\
\hline Immobilized adaptation & $1.25 \pm 0.11 \mathrm{c \#}$ & $1.02 \pm 0.15 \mathrm{cb}$ & $1.00 \pm 0.24 \mathrm{~b} \#$ & $0.84 \pm 0.24 c \#$ & ND \\
\hline Early planktonic adaptation & $1.46 \pm 0.05 b$ & $1.06 \pm 0.05 b$ & $1.22 \pm 0.03 \mathrm{a}$ & $1.22 \pm 0.02 \mathrm{a \#}$ & $0.69 \pm 0.05 a \#$ \\
\hline Biofilm of planktonic adaptation & $1.37 \pm 0.07 \mathrm{a \#}$ & $1.13 \pm 0.09 a \#$ & $1.22 \pm 0.07 a$ & $1.15 \pm 0.06 b \#$ & $0.77 \pm 0.05 \mathrm{c \#}$ \\
\hline NZ3400B & $1.48 \pm 0.04$ & $1.06 \pm 0.04$ & $1.23 \pm 0.03$ & $1.20 \pm 0.03$ & $0.63 \pm 0.07$ \\
\hline
\end{tabular}

${ }^{a}$ The values reported are $\mathrm{OD}_{600 \mathrm{~mm}}$ after $24 \mathrm{~h}$. Values obtained for $\mathrm{NZ3400B}$ represent mean \pm standard deviation from biological triplicates. All other values represent mean \pm standard deviation for all strains recovered from one adaption period, whereas each recovered strain was measured in biological triplicates. Immobilized adaptation, $n=$ 11 strains; early planktonic adaptation, $n=14$ strains; late planktonic adaptation, $n=19$ strains; biofilm of planktonic adaptation, $n=25$ strains. Statistical significance is indicated as follows: a,b,c, significantly different from each other $(P>0.05$ in a paired-sample $t$ test, adjusted for unequal variance or normal distribution when needed); \# significant differences between NZ3400B and L. plantarum groups (one-sample $t$ test, $P \leq 0.05$ ). Abbreviations: ND, not determined; EMS, effluent-MacFarlane-sugar medium.

material). The reference NZ3400B clustered with strains isolated from early planktonic adaptation and clearly separate from strains isolated from effluent and biofilm at the end of adaptation (Fig. 3). Altogether, these results strongly hint toward adaptation of L. plantarum during prolonged cultivation in the gut microbiota. Furthermore, strains did not cluster according to the reactor they were isolated from, suggesting that the adaptation pattern is not dependent on the reactor but rather the time point of isolation.

Phenotypes of these adaptations were stable for at least 190 generations (see Fig. S5 in the supplemental material). This strongly suggests that observed phenotypes are caused by mutations rather than physiological variations.

Mutations in adapted $L$. plantarum strains hint toward adaptive evolution. Stable altered phenotypes of recovered $L$. plantarum strains strongly suggest that these strains harbor mutations. Therefore, whole-genome sequencing of 45 strains randomly selected from adaptations experiments was performed. Comparison to the reference genome NZ3400B revealed 15 strains without any genotypic differences. Out of $18 \mathrm{sin}-$ gle nucleotide polymorphisms (SNPs) confirmed by Sanger sequencing, two were detected in noncoding regions. The remaining 16 SNPs were found in genes involved in signaling, metabolism, transport, and cell surface (Table 2).

The 11 mutated of 12 sequenced strains from the immobilized adaptation belonged to four different genotypes. Among these 11 strains, a mutation in the cell surface protein encoded by LP_RS14255 was found seven times. Further, a mutation in LP_RS14990, encoding a histidine kinase domain, was found three times (Table 2). Eight of $15 \mathrm{~L}$. plantarum strains that were recovered at late stage of the long-term planktonic adaptation were mutated, resulting in five different genotypes (Table 2). Strikingly, an SNP in LP_RS14990 occurred independently during planktonic and immobilized adaptation. Moreover, the SNPs in LP_RS15205 and in the intergenic region between LP_RS05100 and LP_RS05095 were found in strains isolated from two different reactors.

Immobilized adaptation resulted in a bigger fraction of mutated strains, but they consisted predominantly of two isogenic lineages. Planktonic adaptation resulted in less frequent mutagenesis, yet higher mutant diversity. This suggests a difference in adaptation pressure, as already observed for the phenotypic screening. Recovery of some identical mutants from different adaptation experiments suggests that some of the observed mutations are involved in adaptation to the gut microbiota.

Mutations in LP_RS14990 and LP_RS15205 are beneficial for in vitro gut microbiota colonization. The mutation in the histidine kinase protein gene LP_RS14990 and the ROK protein gene LP_RS15205 occurred independently more than once in adaptation experiments. We therefore tested the fitness of each of the mutants $L$. plantarum PA2_06 (C837A in LP_RS15205), IA01 (C979T in LP_RS14990), or PA1.2_01 (C979T in LP_RS14990 and C837A in LP_RS15205) in competition experiments with the reference strain NZ3400B. Ten days of cultivation resulted in an increased abundance of all tested mutants in the gut microbiota compared to NZ3400B (Table 3).

Pyrosequencing indicated that donor 2 gut microbiota had an L. plantarum background. Remarkably, the pyrogram of this background at the position LP_RS15205 


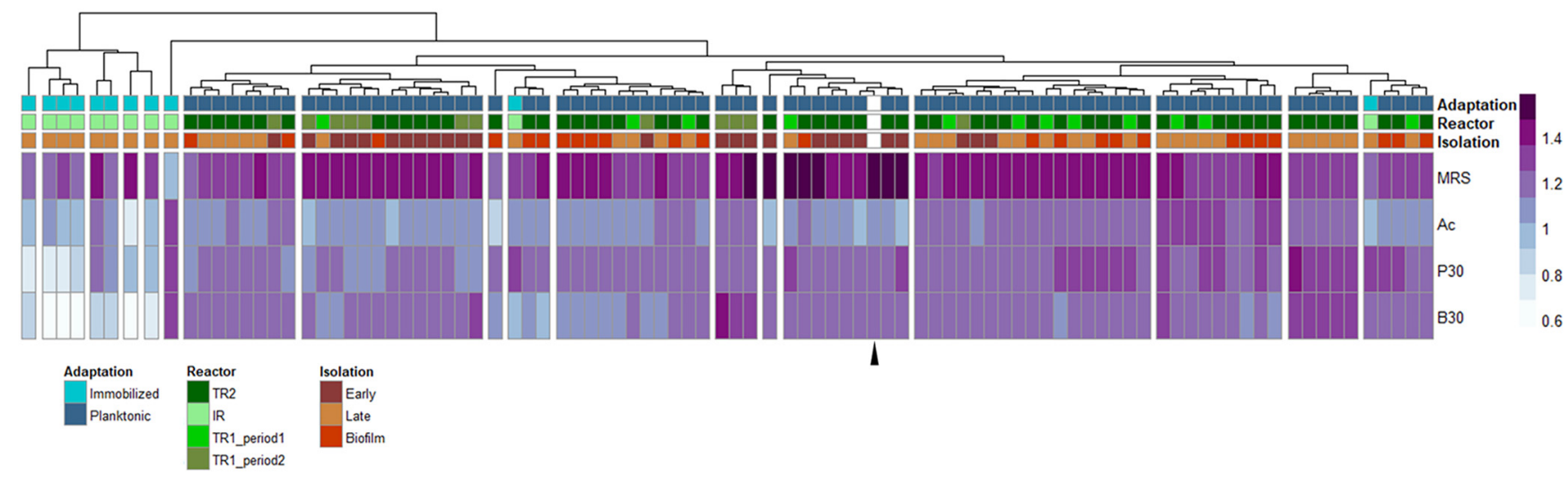

FIG 3 Heatmap visualization of growth behavior of potential mutant L. plantarum strains versus their reference strain NZ3400B in modified MRS medium (single combination of MRS with acetate [50 mM], butyrate [30 mM], or propionate [30 mM]). Symbols are as follows: (i) "Adaptation" indicates strain origin from immobilized or planktonic adaptation trials; (ii) "Reactor" describes the general experiment type for long-term planktonic adaptation (TR2) and the repetition of planktonic adaptation in TR1 (period 1) and TR1 (period 2); (iii) "Isolation" indicates biofilm/time point of isolation (early or late) of a strain during the adaptation cycles. Heatmap colors stand for measured $\Delta \mathrm{OD}_{600 \mathrm{~nm}}$ values after $24 \mathrm{~h}$. The black triangle represents growth of L. plantarum NZ3400B. Ac, acetate; P, propionate; B, butyrate.

C837A was identical to the Pyrogram of a sample containing NZ3400B and PA2_06. This shows that the nucleotide variation of both NZ3400B and PA2_06 also occurs naturally (see Fig. $\mathrm{S} 6$ in the supplemental material).

Mutation C979T in LP_RS14990 is stable under standard culturing conditions. After observing increased fitness of mutants compared to the reference strain, it was investigated whether the mutations of L. plantarum IA01 in LP_RS14990 and L. plantarum PA2_06 in LP_RS15205 are stable during daily repeated batch cultures without the adaptation pressure of the gut microbiota. Stability of the LP_RS14990 mutation in the IA01 strain was observed during 12 batch cultures. However, the mutation C837A in strain PA2_06 was not stable since the reference strain nucleotide reoccurred at $3.5 \% \pm 0.15 \%$ after 12 days (see Table S3 in the supplemental material). Investigation of NZ3400B in repeated MRS batch cultures revealed no occurrence of the SNPs of the mutants.

LP_RS14990 gene replacement in L. plantarum NZ3400B results in delay of gut microbiota colonization. To investigate the role of the LP_RS14990 gene in gut microbiota colonization, a $\Delta$ LP_RS14990 gene replacement strain was constructed and its colonization was compared to NZ3400B. NZ3400B started to colonize the gut microbiota of donor 3 on day 1 and donor 4 at day 3 since levels were above the washout curve (Fig. 4). Levels of $\Delta$ LP_RS14990 in both gut microbiota decreased more rapidly than the washout curve until day 3, suggesting cell death (Fig. 4). Strain $\Delta$ LP_RS14990 started to colonize the gut microbiota of both donors only after 4 days, later than the reference strain.

In silico analysis of LP_RS15205 in L. plantarum. Occurrence of the SNP C837A (L279F) in LP_RS15205 in L. plantarum strains from different reactors and in the background of the gut microbiota suggests an important function of this SNP in survival of L. plantarum in the gut microbiota. In silico analysis of LP_RS15205 revealed that C837A lies in a conserved domain with an ANLxGA motif that is found in Firmicutes and Gammaproteobacteria (data not shown). The function of this domain is unknown, but its conservation throughout two phyla strongly suggests that it might be important for protein function, and the mutation ANLxGA to ANFxGA might have an impact on protein functionality.

\section{DISCUSSION}

Strain improvement is of major importance for food industries and probiotic cultures. Classical evolutionary adaptation approaches focus on specific strain characteristics in abiotic environments like improved performance of starter cultures or lactic acid bacteria $(36,37)$. 


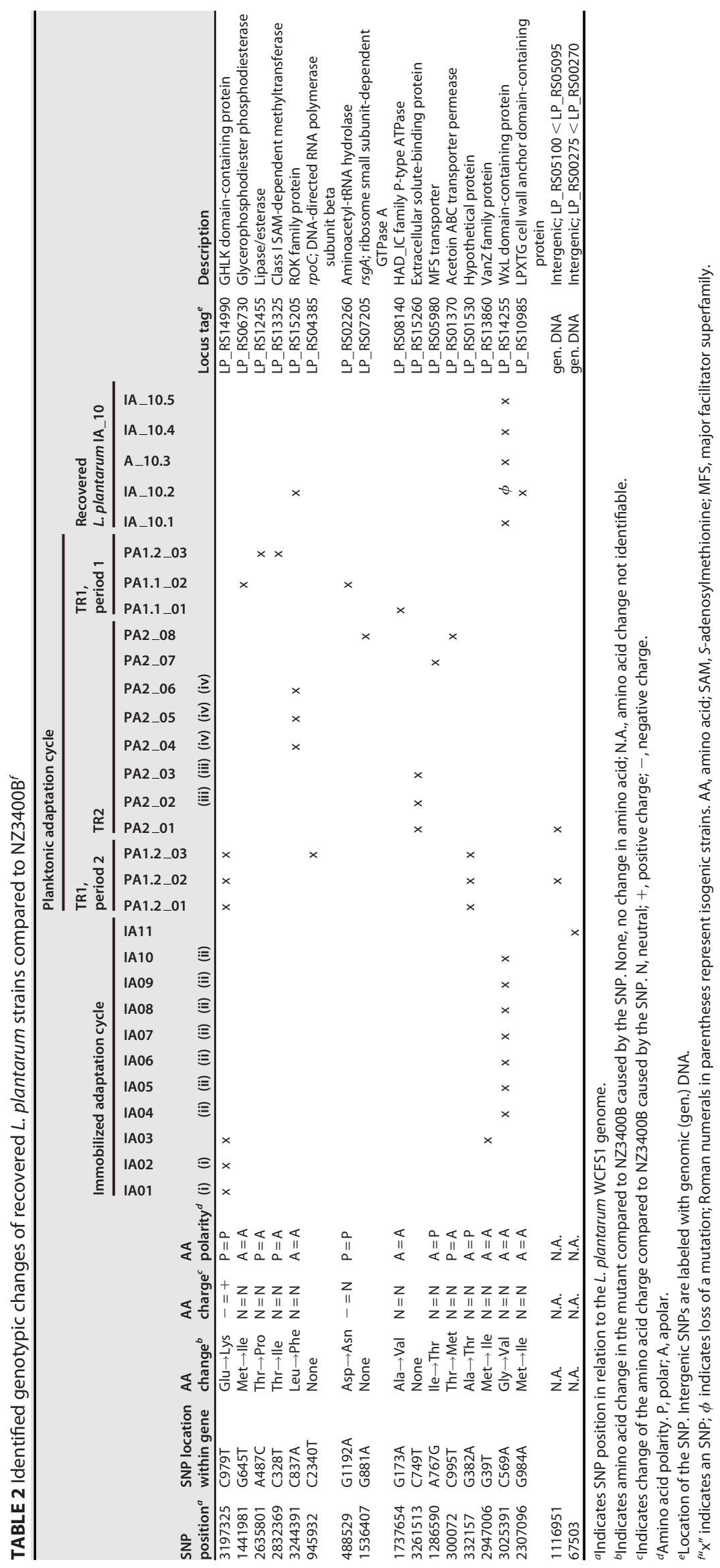


TABLE 3 Increase in relative abundance (in \%) of L. plantarum mutants during 10 days of competition against NZ3400B in in vitro human gut microbiota ${ }^{a}$

\begin{tabular}{lllll}
\hline & & \multicolumn{2}{l}{ L. plantarum mutant strains } & \\
\cline { 3 - 5 } & & IA01 & PA2_06 & PA1.2_01 \\
\hline Donor 2 & Replicate 1 & $38 \pm 5$ & & \\
& Replicate 2 & $25 \pm 1$ & & $11 \pm 2$ \\
Donor 3 & Replicate 1 & $66 \pm 1$ & $26 \pm 3$ & $39 \pm 2$ \\
& Replicate 2 & $51 \pm 1$ & $31 \pm 3$ & \\
Donor 4 & Replicate 1 & & $20 \pm 2$ & \\
& Replicate 2 & & $6 \pm 2$ & \\
\hline
\end{tabular}

aPresented values show the increase of the ratio of $L$. plantarum mutant to the reference strain NZ3400B after 10 days of cultivation compared to the time point of inoculation. Values represent mean \pm standard deviation for three DNA samples isolated at the same time point from the same reactor.

However, for probiotics, improvement of gut colonization needs to consider abiotic and biotic factors. Here, we examined the suitability of the in vitro gut fermentation model PolyFerm $S$ to provide a gut microbiota environment for evolutionary engineering of $L$. plantarum NZ3400 toward a human adult gut microbiota. Immobilization of the donor gut microbiota and cultivation in the PolyFermS allowed reproduction of distinct gut microbiota representative for human adults $(38,39)$. The achieved long-term metabolic and compositional stability allowed the generation of adapted mutants, and the use of several donors increased the external validity of results on observed adaptations. The L. plantarum supplementation method and donor microbiota seem to influence phenotypic and genotypic adaptations.

Even though observed phenotypic differences of recovered L. plantarum were small, $L$. plantarum strains recovered from the immobilized adaptation showed very limited adaptation in contrast to recovered strains from planktonic adaptation trials, although abiotic conditions were highly similar. It thus is assumed that differences in adaptation patterns are caused by the supplementation method. Immobilization protects against stress and entraps cells physically in the system, leading to decreased adaptation pressure (40-42), which is in accordance with our phenotypic screening where strains from immobilized adaptation showed less adaptation. Polymer beads create mucosa-like attachment sites (43), and growth in beads results in a gradual release of sessile cells from the surface that then grow as planktonic cells in the bulk medium (44). The result is a mixed population consisting of cell lineages undergoing various numbers of growth cycles in the planktonic state. This might explain the more diverse phenotypic adaptation pattern among strains from the immobilized adaptation. It was further shown that immobilized cells are genetically more stable than planktonic cells (45-48), which was observed in our study where recovered strains from the immobilized adaptation mainly consisted of two isogenic lineages.

Two observations suggest that the observed genotypic changes in isolated strains were involved in adaptive evolution. The first is the known function of several of the genes affected by SNPs. Activated genes of $L$. plantarum in the murine digestive tract were involved in carbohydrate transport, metabolism and cell surface, sugar-related functions, molecule biosynthesis, and stress response $(49,50)$. Further, exposure of $L$. plantarum to the murine intestinal tract predominantly resulted in mutations in genes encoding cell wall-associated proteins (16). The SNPs identified in our study belong to the categories mentioned above. Remarkably, the latter study identified an SNP in a glycerophosphodiester phosphodiesterase-encoding gene, a gene also affected by an SNP in a recovered strain in our study. In summary, the function of mutated genes is in agreement with previously observed responses of $L$. plantarum to the in vivo intestinal environment. This shows similar selection pressures in the PolyFermS system as in in vivo settings. 

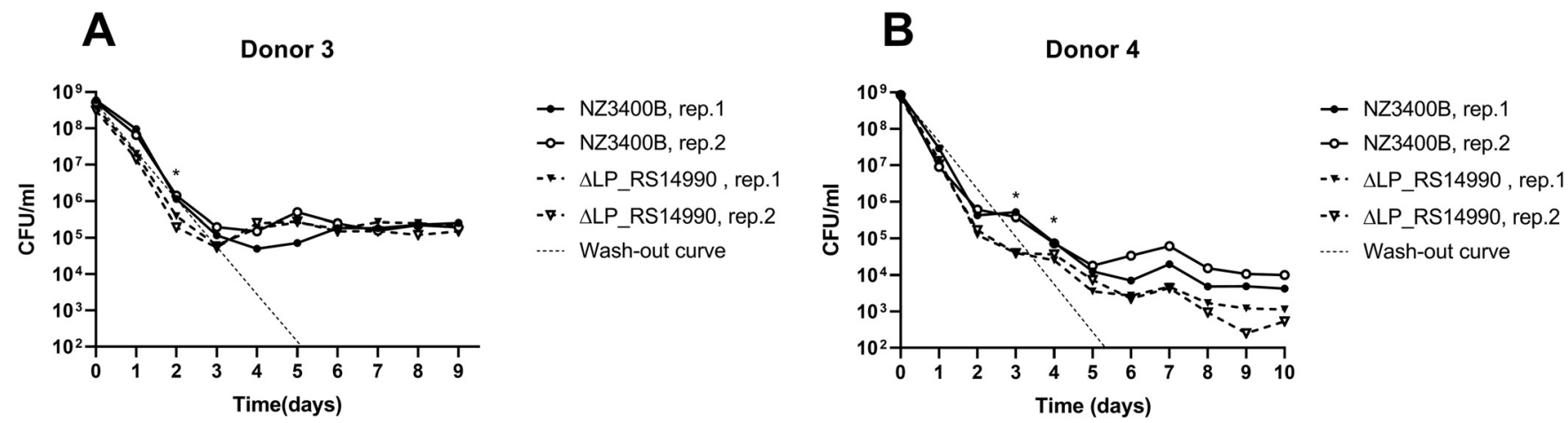

FIG 4 Colonization of $\Delta$ LP_RS14990 compared to the reference strain NZ3400B. L. plantarum strains were added to a level of $10^{9}$ CFU/ml reactor effluent. (A) L. plantarum $\Delta$ LP_RS14990 and NZ3400B were added into each of the two reactors (rep.1, rep.2) in donor 3 gut microbiota. (B) L. plantarum $\Delta$ LP_RS14990 and NZ3400B were added into each of the two reactors (rep.1, rep.2) in donor 4 gut microbiota. Dotted lines indicate the theoretical washout curve of the system. Cell count is depicted on the $y$ axis, and days of cultivation in the microbiota are shown on the $x$ axis, where 0 corresponds to the day of $L$. plantarum supplementation. *, significantly different colonization levels between NZ3400B and $\Delta$ LP_RS14990 ( $P \leq 0.05$, paired-sample $t$ test).

The second observation indicating adaptive evolution in our experiment is that three mutations were found independently in multiple experiments. Since mutations occur continuously, $-80^{\circ} \mathrm{C}$ stock cultures are rarely isogenic, and the mutants found in our study could have been already present as subculture in the NZ3400 stock (16). To counteract this bias, two different stock cultures, NZ3400 and NZ3400B were used for the immobilized and planktonic adaptation, respectively. Occurrence of identical mutants recovered from immobilized and planktonic adaptation with the SNP in LP_RS14990 seems therefore unlikely to originate from a stock subculture, as confirmed by Sanger sequencing (data not shown). Furthermore, the mutation was not detected by pyrosequencing of NZ3400B before inoculation (data not shown), minimizing a role for subcultures in our experiments. Moreover, the increased fitness of mutants shows the beneficial effect of detected mutations. This strongly supports that observed mutations are caused by adaptive evolution and demonstrates the suitability of the PolyFermS model to select relevant mutations related to the human gut microbiota. The exact nature of the evolutionary pressure in our system is not known and remains to be elucidated. It is likely the result of a combination of factors such as the competition for nutrients, metabolic cross-feeding, physiochemical factors, or presence of antimicrobial metabolites.

In conclusion, we demonstrated successful application of the continuous PolyFerms gut fermentation model to provide a long-term stable gut microbiota to generate adapted mutants to this environment. Immobilization of strains not only allows adaptive evolution of noncolonizers but also creates a culture consisting of sessile and planktonic cells mimicking the human gastrointestinal tract. The conditions of the model can be easily changed to other needs and selective pressures, including the source of microbiota and abiotic conditions. This novel technology enables identification of genes involved in gut microbiota colonization, persistence, and metabolism. The PolyFerms system could further be designed and applied for different fermentations, to trace and identify evolutionary and ecological processes between an exogenous single strain and a complex ecosystem.

\section{MATERIALS AND METHODS}

Bacterial strains and growth conditions. Bacterial strains used during this study are listed in Table S1 in the supplemental material. L. plantarum NZ3400 (51) was used as reference strain. NZ3400 is a derivative of WCFS1, harboring a CM resistance cassette $\left(\mathrm{P}_{32}\right.$-cat $)$ in a neutral locus on the chromosome. L. plantarum was grown in De Man, Rogosa, and Sharpe (MRS; Labo-Life Sàrl, Pully, Switzerland) broth at $37^{\circ} \mathrm{C}$, overnight. L. plantarum viable cells were enumerated by plating on MRS agar supplemented with $\mathrm{CM}(10 \mu \mathrm{g} / \mathrm{ml})$, with aerobic incubation at $37^{\circ} \mathrm{C}$, overnight.

Immobilization of adult fecal microbiota. Fecal samples were obtained from four healthy adult individuals ( 27 to 31 years old) who did not take antibiotics and probiotics for at least 3 months and did not show detectable microbial growth on MRS +CM plates to avoid interference with L. plantarum NZ3400 recovery. Out of 15 tested fecal samples, four samples that did not show microbial growth on MRS+CM were chosen for fermentation (donors 1 to 4). The Ethics Committee of ETH Zürich exempted 
this study from review because the sample collection procedure has not been performed under conditions of intervention. Informed written consent was obtained from fecal donors. Fecal samples were immediately transferred to an anaerobic chamber within $2 \mathrm{~h}$ after defecation and suspended at $20 \%$ (wt/ vol) in reduced peptone water $(0.1 \%, \mathrm{pH} 7$; Thermo Fisher Diagnostics AG, Pratteln, Switzerland). Immobilization of $10 \mathrm{ml}$ fecal slurry in polymer gel beads (gellan gum [2.5\%, wt/vol], xanthan [0.25\%, wt/ vol], and sodium citrate $[0.2 \%$, wt/vol]) was performed as previously described $(21,44)$. The inoculum bioreactor (IR) (Sixfors; Infors, Bottmingen, Switzerland) was filled with $140 \mathrm{ml}$ of vitamin-supplemented MacFarlane medium (Text S1), formulated to mimic the chyme entering the colon $(20,52)$, and inoculated with $60 \mathrm{ml}$ of fecal gel beads. Beads were colonized in two fed-batch fermentations carried out at $37^{\circ} \mathrm{C}, \mathrm{pH} 5.8$, by controlled addition of $\mathrm{NaOH}(2.5 \mathrm{M})$, stirring at $180 \mathrm{rpm}$, and replacing $100 \mathrm{ml}$ of spent medium with fresh medium after $16 \mathrm{~h}(21)$. Anaerobiosis was set by purging the bioreactor headspace with $\mathrm{CO}_{2}$ and monitored by redox potential probes.

Proximal colon fermentation in the PolyFerms model using immobilized human gut microbiota. Continuous proximal colonic in vitro fermentations with immobilized human adult gut microbiota were performed in bioreactors as reported previously $(26,53)$. All fermentations were operated to mimic the adult proximal colon as described above for batch fermentation. Fresh medium was continuously added to the IR $(25 \mathrm{ml} / \mathrm{h})$, and fermented medium was removed to maintain a working volume of $200 \mathrm{ml}$. Because short-chain fatty acids (SCFAs) are the main fermentation end products of the gut microbiota, their stable production is a convenient, easily measurable marker for stability of continuous intestinal fermentation models $(21,54)$. The IR was run in continuous mode for at least 10 days to reach metabolic stability indicated by lower than $10 \%$ day-to-day variation $(25,54)$ in SCFA concentration, before connecting second-stage TRs. TRs were inoculated by the IR at $1.25 \mathrm{ml} / \mathrm{h}$ and fed at $23.75 \mathrm{ml} / \mathrm{h}$. TRs were operated continuously for 4 days prior to L. plantarum supplementation in order to establish a gut microbiota activity akin to the IR. Addition of planktonic or immobilized $L$. plantarum was tested in IR and TRs with different donor microbiota (Fig. 1). In the case of two successive treatment periods in the same reactor, the reactor was disconnected after the first treatment period, sterilized, reconnected, and stabilized for 4 days before starting a second treatment.

Reactor effluent samples were taken daily to monitor the fermentation process, centrifuged for $10 \mathrm{~min}$ at $14,000 \times \mathrm{g}, 4^{\circ} \mathrm{C}$, and stored at $-20^{\circ} \mathrm{C}$. Pellets were used for DNA extraction, and supernatants were used for metabolite analysis.

Adaptive evolutionary engineering of $L$. plantarum. (i) Adaptive evolution using immobilized $L$. plantarum NZ3400. Adaptive evolution of immobilized L. plantarum NZ3400 was tested in a continuously run single-stage IR (IR1) inoculated with donor 1 fecal beads (Fig. 1A). An L. plantarum overnight culture was harvested at $4^{\circ} \mathrm{C}, 4,000 \times g$, for $10 \mathrm{~min}$ and washed twice in phosphate-buffered saline (PBS), pH 6.2. Immobilization of L. plantarum was done as described for fecal samples under aerobic conditions. Beads were colonized during two $\mathrm{pH}$-controlled batch cultures at $37^{\circ} \mathrm{C}$ for $16 \mathrm{~h}$ with stirring at $150 \mathrm{rpm}$. Colonized beads were washed in PBS, supplemented with cryoprotective buffer (55), and stored at $-80^{\circ} \mathrm{C}$. Before use, L. plantarum beads were reactivated during two batch cultures as described for colonization and washed twice in PBS. L. plantarum viable cell counts were determined by crushing 1 $\mathrm{g}$ of beads in PBS with a spatula and plating serial dilutions. Four grams of beads $\left(5 \times 10^{9} \mathrm{CFU}\right.$ L. plantarum/g) was added to the single-stage IR1 and cultivated for 53 days.

(ii) Adaptive evolution using planktonic $L$. plantarum NZ3400B. Due to microbial growth on MRS +CM plates of donor 1 fecal sample at this time, a new donor was chosen for the planktonic adaptation. To prevent carryover of possible mutants present in the initial NZ3400 stock culture, a new stock from a single NZ3400 colony isolate, designated NZ3400B was produced. NZ3400B was subjected to PacBio sequencing and used for all subsequent planktonic supplementation trials. NZ3400 and NZ3400B differed in eight SNPs but none thereof in SNP-affected genes of recovered $L$. plantarum. Adaptation of planktonic $L$. plantarum NZ3400B was tested in TRs continuously inoculated by the effluent from IR2 containing beads with immobilized fecal microbiota of donor 2 (Fig. 1B). Because the PolyFerms model was built with six TRs, two consecutive treatment periods were performed to test all treatments. L. plantarum strains were grown overnight, harvested, washed twice in PBS, resuspended in MacFarlane medium, and added to the TRs to a final level of $10^{9} \mathrm{CFU}$ L. plantarum/ml effluent. Long-term planktonic adaptation was tested in TR2 operated for 72 days and repeated in TR1 (period 1) and TR1 (period 2) for 44 and 23 days, respectively (Fig. 1B).

Furthermore, the strain IA10, recovered from the immobilized adaptation, was added in planktonic state to TR3 (period 1) and TR3 (period 2) containing donor 2 gut microbiota to investigate effects of adaptations that occurred during the immobilized adaptation (Fig. 1B).

Metabolite analysis of the continuous colon fermentation. Reactors were sampled daily for analysis of the SCFAs acetate, butyrate, and propionate; branched-chain fatty acids isobutyrate, isovalerate, and valerate; and intermediate metabolites lactate and formate (56). Concentrations were determined by high-performance liquid chromatography (HPLC) as described previously (23).

Microbial profiling by $16 \mathrm{~S}$ rRNA gene amplicon sequencing. Genomic DNA of fecal and effluent samples was extracted using the FastDNA Spin kit for soil (MP Biomedicals, Illkirch, France) according to the manufacturer's instructions. The V4 region of the $16 \mathrm{~S}$ rRNA gene was amplified with the primers 806R (5'-GGACTACHVGGGTWTCTAAT-3') and 515F (5'-GTGCCAGCMGCCGCGGTAA-3'). Amplicons were barcoded PCR based. Library preparation and sequencing (Illumina, CA, USA) using an Illumina MiSeq flow cell with a V2 reagent kit for $2 \times 250$-bp paired-end Nextera chemistry supplemented with $10 \%$ PhiX were performed in collaboration with the Genetic Diversity Center (GDC; ETH Zürich, Switzerland).

Raw data obtained from $16 \mathrm{~S}$ rRNA sequencing were processed using Cutadapt (57) and DADA2 pipeline (58) to obtain amplicon sequence variants. Taxonomy was assigned using the SILVA database (v.132) (59) (full method described in Text S1). 
Recovery of $L$. plantarum from the gut microbiota. L. plantarum colonization was determined by plating on MRS +CM agar. The combination of the MRS selectivity for lactobacilli and enterococci together with aerobe incubation and presence of antibiotics allowed growth repression of all other bacteria. Data were compared to the theoretical washout curve determined for absence of growth, from the following equation: $c_{t}=c_{0} \times e^{(t / R T)}(53)$, where $c_{0}$ and $c_{t}$ represent cell concentration at time point zero and $t$, respectively, and RT corresponds to the retention time. Colonies were randomly picked, incubated in MRS+CM overnight, mixed 1:1 with 60\% (vol/vol) glycerol (Sigma-Aldrich Chemie GmbH, Buchs, Switzerland), and stored at $-80^{\circ} \mathrm{C}$.

Natural biofilm formed in TR2, used for long-term planktonic adaptation, and the repetition experiment in TR1 (period 1). To recover L. plantarum from biofilms, the vessels were emptied and washed twice with PBS. Remaining biofilm was removed and homogenized with glass beads $(5 \mathrm{~mm}$; VWR International AG, Dietikon, Switzerland) in dilution solution containing $0.85 \%(\mathrm{wt} / \mathrm{vol}) \mathrm{NaCl}$ and $0.1 \%(\mathrm{wt} / \mathrm{vol})$ peptone from casein (VWR International AG, Dietikon, Switzerland). Dilutions were plated with subsequent strain recovery and storage performed as described above.

Phenotypic characterization of recovered $L$. plantarum strains. Growth behavior of recovered $L$. plantarum strains was analyzed in MRS supplemented with each of the main SCFAs of the human gut microbiota, acetate $(50,75$, and $100 \mathrm{mM})$, butyrate $(15,30$, and $45 \mathrm{mM})$, and propionate $(15,30$, and $45 \mathrm{mM}$ ) in similar concentrations as measured during colonic fermentations (see Fig. S2 in the supplemental material). The abiotic gut fermentation environment was simulated in effluent-MacFarlane-sugar (EMS) medium consisting of filter-sterilized PolyFermS effluent, MacFarlane medium in a 9:1 ratio, and $0.75 \%$ (wt/vol) glucose (see Fig. S1 in the supplemental material). Glucose was added since L. plantarum was unable to grow in MacFarlane medium. For comparison of the effect on adaption in immobilized and long-term planktonic adaptation trials performed in TR2 (Fig. 1B), recovered strains were divided into four groups based on their origin of isolation: (i) 11 strains from the effluent at the late stage of immobilized adaptation after 53 days, (ii) 14 strains from the effluent during day 7 and day 23 (early planktonic adaptation), (iii) 19 strains from the effluent during day 60 and day 72 (late planktonic adaptation), and (iv) 25 strains from the biofilm of planktonic adaptation after 72 days. Strains were isolated at an early stage after seven (stable L. plantarum colonization) and 23 days and a late stage of 60 and 72 days to increase the chance to observe adaptation. Biofilm was sampled on the last day of fermentation because the reactor had to be emptied for biofilm sampling. Growth analysis was done in $96-$ well tissue culture test plates (Bioswisstec AG, Schaffhausen, Switzerland). Wells were filled with $200 \mu \mathrm{l}$ of medium and inoculated with the potentially adapted L. plantarum at $37^{\circ} \mathrm{C}$. Growth was monitored by optical density (OD) measurement at $600 \mathrm{~nm}$ in a plate reader after $24 \mathrm{~h}$ (PowerWaveTMXS; Bio-Tek Instrument Inc., Winooski, VT, USA) in biological triplicates.

Phenotype stability was assessed by repeated daily culturing of strains in MRS for 28 days, approximately 190 generations, as presented above. Stability was measured after 1, 7, 14, 21, and 28 days, which corresponds to the time of transcriptome homogenization among $L$. plantarum strains isolated from different habitats (60).

Complete genome sequencing and data analysis. L. plantarum genomic DNA was isolated via lysozyme-based cell lysis (61) followed by purification using the Wizard Genomic DNA purification kit according to the manufacturer's instructions (Promega, Dübendorf, Switzerland). The genome of the reference strain NZ3400B was sequenced at the Functional Genomics Center Zurich (Zürich, Switzerland) on PacBio RS II (Pacific Biosciences, Menlo Park, CA, USA) using one SMRT cell. Reads were assembled using Hierarchical Genome Assembly Process (HGAP) assembly as described previously (62). All other $L$. plantarum strains were sequenced using Illumina MiniSeq (Illumina, CA, USA) with 250-bp paired-end reads at the Institute for Food Safety and Hygiene, University of Zurich (62). Reads were merged and mapped to the reference genome L. plantarum NZ3400B using CLC Genomic Workbench 11.0 (Qiagen CLC bio, Aarhus, Denmark) using default parameters. Single nucleotide polymorphisms (SNPs) were extracted using Parsnp (63). SNPs were confirmed by Sanger Sequencing (Eurofins GATC, Biotech GmbH, Constance, Germany). SNP-related changes in amino acid sequence were determined in CLC Genomic Workbench.

Competition experiments in the human gut microbiota. Competition experiments between NZ3400B and the mutant strains L. plantarum IA01, PA1.2_01, and PA2_06 were performed to determine the effect of the mutations on gut microbiota colonization (Fig. 1B). NZ3400B was paired with each of the three mutant strains in a 1:1 ratio to reach $10^{9} \mathrm{CFU} / \mathrm{ml}$ reactor effluent in the gut microbiota and cultivated for 10 days. Ten days was sufficient to obtain stable L. plantarum colonization for at least 4 days but also as briefly as possible to minimize the chance of proliferation of new mutants. Relative abundance of the strains in the complex community was determined by measuring allele frequency of the genes LP_RS14990 and LP_RS15205, respectively, via Pyrosequencing (full method described in Text S1).

Stability of mutations under standard cultivation conditions. Stability of the mutations in L. plantarum IA01 and PA2_06 was investigated during repeated daily cultures in MRS for 12 days, approximately 81 generations, performed in triplicates. Cultivation of NZ3400B served as control. Allele frequency was determined via pyrosequencing as described above.

Plasmid construction and LP_RS14990 gene replacement of $L$. plantarum NZ3400B. Six mutants carrying the mutation in LP_RS14990 were recovered from independent adaptation experiments. To investigate the involvement of this gene in gut microbiota colonization, an L. plantarum $\triangle$ LP_RS14990 knockout was constructed by double-crossover gene replacement in L. plantarum NZ3400B (full method in Text S1). The knockout strain was subsequently added to the gut microbiota, and colonization levels were compared to the reference strain NZ3400B (Fig. 1B). 
In silico analysis of LP_RS15205 in L. plantarum. LP_RS15205 was affected by an identical mutation in four strains recovered from two independent adaptation experiments. Since this mutation was also found in the background gut microbiota, in silico analysis of this locus was performed. Complete genome sequences of Firmicutes $(n=557)$, Bacteroidetes $(n=218)$, Actinomycetes $(n=457)$, and Gammaproteobacteria $(n=638)$ from the NCBI genome database were downloaded in May 2020. The amino acid sequence of LP_RS15205 was subjected to a BLAST search against these genomes using standard settings, and significant hits were aligned using MUSCLE (64).

Data analysis. Statistics for growth experiments were calculated in R (version 3.6.2) using a onesample $t$ test for comparison to $L$. plantarum NZ3400B and a paired-sample $t$ test for comparison within recovered $L$. plantarum groups. Values represent mean values \pm standard deviations. The heatmap was generated using the $\mathrm{R}$ pheatmap package and Euclidean distance measure. Allele frequency determination by pyrosequencing was calculated based on three extracted DNA samples of the same time point. Graphs were created using GraphPad Prism version 8 (GraphPad Software Inc., San Diego, CA, USA).

\section{SUPPLEMENTAL MATERIAL}

Supplemental material is available online only.

TEXT S1, DOCX file, $0.02 \mathrm{MB}$.

FIG S1, TIF file, $0.03 \mathrm{MB}$.

FIG S2, TIF file, $0.1 \mathrm{MB}$.

FIG S3, TIF file, $0.1 \mathrm{MB}$.

FIG S4, TIF file, 0.2 MB.

FIG S5, TIF file, $0.04 \mathrm{MB}$.

FIG S6, TIF file, $0.1 \mathrm{MB}$.

TABLE S1, DOCX file, $0.02 \mathrm{MB}$.

TABLE S2, DOCX file, $0.01 \mathrm{MB}$.

TABLE S3, DOCX file, $0.01 \mathrm{MB}$.

\section{ACKNOWLEDGMENTS}

We thank Alfonso Die for assistance during HPLC measurements, Florentin Constancias for his support converting $16 \mathrm{~S}$ sequencing raw data into amplicon sequence variants, and Julia Iselin for experimental assistance. Pyrosequencing was set up in collaboration with the Genetic Diversity Centre (GDC), ETH Zurich.

The study was funded by the ETH Zurich research grant program (ETH-42 16-1).

\section{REFERENCES}

1. Ross RP, Morgan S, Hill C. 2002. Preservation and fermentation: past, present and future. Int J Food Microbiol 79:3-16. https://doi.org/10.1016/ s0168-1605(02)00174-5.

2. Demain AL, Adrio JL. 2008. Contributions of microorganisms to industrial biology. Mol Biotechnol 38:41-55. https://doi.org/10.1007/s12033-007-0035-z.

3. Cleveland J, Montville TJ, Nes IF, Chikindas ML. 2001. Bacteriocins: safe, natural antimicrobials for food preservation. Int J Food Microbiol 71:1-20. https://doi.org/10.1016/s0168-1605(01)00560-8.

4. Hill C, Guarner F, Reid G, Gibson GR, Merenstein DJ, Pot B, Morelli L, Canani RB, Flint HJ, Salminen S, Calder PC, Sanders ME. 2014. Expert consensus document: The International Scientific Association for Probiotics and Prebiotics consensus statement on the scope and appropriate use of the term probiotic. Nat Rev Gastroenterol Hepatol 11:506-514. https://doi .org/10.1038/nrgastro.2014.66.

5. Butler PR, Brown M, Oliver SG. 1996. Improvement of antibiotic titers from Streptomyces bacteria by interactive continuous selection. Biotechnol Bioeng 49:185-196. https://doi.org/10.1002/(SICI)1097-0290(19960120)49:2<185::AID -BIT7>3.0.CO;2-M.

6. Dragosits M, Mattanovich D. 2013. Adaptive laboratory evolution - principles and applications for biotechnology. Microb Cell Fact 12:64. https:// doi.org/10.1186/1475-2859-12-64.

7. Sauer U. 2001. Evolutionary engineering of industrially important microbial phenotypes. Adv Biochem Eng Biotechnol 73:129-169. https://doi .org/10.1007/3-540-45300-8_7.

8. Bachmann H, Pronk JT, Kleerebezem M, Teusink B. 2015. Evolutionary engineering to enhance starter culture performance in food fermentations. Curr Opin Biotechnol 32:1-7. https://doi.org/10.1016/j.copbio.2014.09.003.

9. Mans R, Daran JG, Pronk JT. 2018. Under pressure: evolutionary engineering of yeast strains for improved performance in fuels and chemicals production. Curr Opin Biotechnol 50:47-56. https://doi.org/10.1016/j .copbio.2017.10.011.

10. Cakar ZP, Turanlı-Yıldız B, Alkım C, Yılmaz Ü. 2012. Evolutionary engineering of Saccharomyces cerevisiae for improved industrially important properties. FEMS Yeast Res 12:171-182. https://doi.org/10.1111/j.1567-1364.2011.00775.x.

11. Winkler JD, Kao KC. 2014. Recent advances in the evolutionary engineering of industrial biocatalysts. Genomics 104:406-411. https://doi.org/10 .1016/j.ygeno.2014.09.006.

12. Bachmann H, Starrenburg MJC, Molenaar D, Kleerebezem M, Vlieg JETV. 2012. Microbial domestication signatures of Lactococcus lactis can be reproduced by experimental evolution. Genome Res 22:115-124. https:// doi.org/10.1101/gr.121285.111.

13. Conrad TM, Frazier M, Joyce AR, Cho BK, Knight EM, Lewis NE, Landick R, Palsson BO. 2010. RNA polymerase mutants found through adaptive evolution reprogram Escherichia coli for optimal growth in minimal media. Proc Natl Acad Sci U S A 107:20500-20505. https://doi.org/10.1073/pnas 0911253107.

14. Lee JW, Lee SY, Yoo JS. 2010. Metabolic and evolutionary engineering of Mannheimia succiniciproducens for the enhanced succinate productivity. J Biotechnol 150(Suppl):516. https://doi.org/10.1016/j.jbiotec.2010.09.821.

15. Roca C, Albuquerque G, Reis M. 2010. Evolutionary engineering of Actinobacillus succinogenes for improved succinic acid production on glycerol. J Biotechnol 150(Suppl):373. https://doi.org/10.1016/j.jbiotec.2010.09.451.

16. van Bokhorst-van de Veen $H$, Smelt MJ, Wels $M$, van Hijum SAFT, de Vos $P$, Kleerebezem M, Bron PA. 2013. Genotypic adaptations associated with prolonged persistence of Lactobacillus plantarum in the murine digestive tract. Biotechnol J 8:895-904. https://doi.org/10.1002/biot.201200259.

17. Lesker TR, Durairaj AC, Galvez EJC, Lagkouvardos I, Baines JF, Clavel T, Sczyrba A, McHardy AC, Strowig T. 2020. An integrated metagenome 
catalog reveals new insights into the murine gut microbiome. Cell Rep 30:2909-2922.e6. https://doi.org/10.1016/j.celrep.2020.02.036.

18. Lagkouvardos I, Pukall R, Abt B, Foesel BU, Meier-Kolthoff JP, Kumar N, Bresciani A, Martinez I, Just S, Ziegler C, Brugiroux S, Garzetti D, Wenning M, Bui TPN, Wang J, Hugenholtz F, Plugge CM, Peterson DA, Hornef M, Baines JF, Smidt H, Walter J, Kristiansen K, Nielsen HB, Haller D, Overmann J, Stecher B, Clavel T. 2016. The Mouse Intestinal Bacterial Collection (miBC) provides host-specific insight into cultured diversity and functional potential of the gut microbiota. Nat Microbiol 1:16219. https://doi .org/10.1038/nmicrobiol.2016.219.

19. Pham VT, Mohajeri MH. 2018. The application of in vitro human intestinal models on the screening and development of pre- and probiotics. Benef Microbes 9:725-742. https://doi.org/10.3920/BM2017.0164.

20. Macfarlane GT, Macfarlane S, Gibson GR. 1998. Validation of a three-stage compound continuous culture system for investigating the effect of retention time on the ecology and metabolism of bacteria in the human colon. Microb Ecol 35:180-187. https://doi.org/10.1007/s002489900072.

21. Fehlbaum S, Chassard C, Haug MC, Fourmestraux C, Derrien M, Lacroix C. 2015. Design and investigation of PolyFermS in vitro continuous fermentation models inoculated with immobilized fecal microbiota mimicking the elderly colon. PLoS One 10:e0142793. https://doi.org/10.1371/journal .pone.0142793.

22. Doo EH, Chassard C, Schwab C, Lacroix C. 2017. Effect of dietary nucleosides and yeast extracts on composition and metabolic activity of infant gut microbiota in PolyFerms colonic fermentation models. FEMS Microbiol Ecol 93(8). https://doi.org/10.1093/femsec/fix088.

23. Tanner SA, Zihler Berner A, Rigozzi E, Grattepanche F, Chassard C, Lacroix C. 2014. In vitro continuous fermentation model (PolyFermS) of the swine proximal colon for simultaneous testing on the same gut microbiota. PLoS One 9:e94123. https://doi.org/10.1371/journal.pone.0094123.

24. Poeker SA, Lacroix C, de Wouters T, Spalinger MR, Scharl M, Geirnaert A. 2019. Stepwise development of an in vitro continuous fermentation model for the murine caecal microbiota. Front Microbiol 10:1166. https:// doi.org/10.3389/fmicb.2019.01166.

25. Asare PT. 2019. Bioprotective effects of reuterin produced by bioconversion of glycerol by Lactobacillus reuteri in fresh-cut vegetable and chicken gut. Dissertation ETH no. 26433. ETH Zurich, Zurich, Switzerland.

26. Berner AZ, Fuentes S, Dostal A, Payne AN, Gutierrez PV, Chassard C, Grattepanche F, de Vos WM, Lacroix C. 2013. Novel polyfermentor intestinal model (PolyFermS) for controlled ecological studies: validation and effect of pH. PLoS One 8:e77772. https://doi.org/10.1371/journal.pone .0077772 .

27. Payne AN, Zihler A, Chassard C, Lacroix C. 2012. Advances and perspectives in in vitro human gut fermentation modeling. Trends Biotechnol 30:17-25. https://doi.org/10.1016/j.tibtech.2011.06.011.

28. Bircher L, Schwab C, Geirnaert A, Greppi A, Lacroix C. 2020. Planktonic and sessile artificial colonic microbiota harbor distinct composition and reestablish differently upon frozen and freeze-dried long-term storage. mSystems 5:e00521-19. https://doi.org/10.1128/mSystems.00521-19.

29. De Weirdt R, Van de Wiele T. 2015. Micromanagement in the gut: microenvironmental factors govern colon mucosal biofilm structure and functionality. NPJ Biofilms Microbiomes 1:15026. https://doi.org/ 10.1038/npjbiofilms.2015.26.

30. Sonnenburg JL, Angenent LT, Gordon Jl. 2004. Getting a grip on things: how do communities of bacterial symbionts become established in our intestine? Nat Immunol 5:569-573. https://doi.org/10.1038/ni1079.

31. Quintana MCD, García PG, Fernández AG. 1999. Establishment of conditions for green table olive fermentation at low temperature. Int J Food Microbiol 51:133-143. https://doi.org/10.1016/s0168-1605(99)00123-3.

32. Enan G, El-Essawy AA, Uyttendaele M, Debevere J. 1996. Antibacterial activity of Lactobacillus plantarum UG1 isolated from dry sausage: characterization, production and bactericidal action of plantaricin UG1. Int J Food Microbiol 30:189-215. https://doi.org/10.1016/0168-1605(96)00947-6.

33. Ahrné S, Nobaek S, Jeppsson B, Adlerberth I, Wold AE, Molin G. 1998. The normal Lactobacillus flora of healthy human rectal and oral mucosa. J Appl Microbiol 85:88-94. https://doi.org/10.1046/j.1365-2672.1998.00480.x.

34. Kleerebezem M, Boekhorst J, van Kranenburg R, Molenaar D, Kuipers OP, Leer R, Tarchini R, Peters SA, Sandbrink HM, Fiers MW, Stiekema W, Lankhorst RM, Bron PA, Hoffer SM, Groot MN, Kerkhoven R, de Vries M, Ursing B, de Vos WM, Siezen RJ. 2003. Complete genome sequence of Lactobacillus plantarum WCFS1. Proc Natl Acad Sci U S A 100:1990-1995. https://doi.org/10.1073/pnas.0337704100.

35. van den Nieuwboer M, van Hemert S, Claassen E, de Vos WM. 2016. Lactobacillus plantarum WCFS1 and its host interaction: a dozen years after the genome. Microb Biotechnol 9:452-465. https://doi.org/10.1111/1751 -7915.12368 .

36. Johansen E. 2018. Use of natural selection and evolution to develop new starter cultures for fermented foods. Annu Rev Food Sci Technol 9:411-428. https://doi.org/10.1146/annurev-food-030117-012450.

37. Wang C, Cui Y, Qu X. 2018. Mechanisms and improvement of acid resistance in lactic acid bacteria. Arch Microbiol 200:195-201. https://doi.org/ 10.1007/s00203-017-1446-2.

38. Arumugam M, Raes J, Pelletier E, Le Paslier D, Yamada T, Mende DR, Fernandes GR, Tap J, Bruls T, Batto JM, Bertalan M, Borruel N, Casellas F, Fernandez L, Gautier L, Hansen T, Hattori M, Hayashi T, Kleerebezem M, Kurokawa K, Leclerc M, Levenez F, Manichanh C, Nielsen HB, Nielsen T, Pons N, Poulain J, Qin J, Sicheritz-Ponten T, Tims S, Torrents D, Ugarte E, Zoetendal EG, Wang J, Guarner F, Pedersen O, de Vos WM, Brunak S, Dore J, MetaHIT Consortium, Antolin M, Artiguenave F, Blottiere HM, Almeida M, Brechot C, Cara C, Chervaux C, Cultrone A, Delorme C, Denariaz G, Dervyn $R$, et al. 2011. Enterotypes of the human gut microbiome. Nature 473:174-180. https://doi.org/10.1038/nature09944.

39. Segata N, Haake SK, Mannon P, Lemon KP, Waldron L, Gevers D, Huttenhower C, Izard J. 2012. Composition of the adult digestive tract bacterial microbiome based on seven mouth surfaces, tonsils, throat and stool samples. Genome Biol 13:R42. https://doi.org/10.1186/gb-2012-13-6 $-r 42$.

40. Spoering AL, Lewis K. 2001. Biofilms and planktonic cells of Pseudomonas aeruginosa have similar resistance to killing by antimicrobials. J Bacteriol 183:6746-6751. https://doi.org/10.1128/JB.183.23.6746-6751.2001.

41. Knudsen GM, Nielsen MB, Grassby T, Danino-Appleton V, Thomsen LE, Colquhoun IJ, Brocklehurst TF, Olsen JE, Hinton JCD. 2012. A third mode of surface-associated growth: immobilization of Salmonella enterica serovar Typhimurium modulates the RpoS-directed transcriptional programme. Environ Microbiol 14:1855-1875. https://doi.org/10.1111/j.1462 -2920.2012.02703.x.

42. Skandamis PN, Gounadaki AS, Geornaras I, Sofos JN. 2012. Adaptive acid tolerance response of Listeria monocytogenes strains under planktonic and immobilized growth conditions. Int J Food Microbiol 159:160-166. https://doi.org/10.1016/j.ijfoodmicro.2012.07.027.

43. Walker AW, Duncan SH, Harmsen HJM, Holtrop G, Welling GW, Flint HJ. 2008. The species composition of the human intestinal microbiota differs between particle-associated and liquid phase communities. Environ Microbiol 10:3275-3283. https://doi.org/10.1111/j.1462-2920.2008.01717.x.

44. Cinquin C, Le Blay G, Fliss I, Lacroix C. 2004. Immobilization of infant fecal microbiota and utilization in an in vitro colonic fermentation model. Microb Ecol 48:128-138. https://doi.org/10.1007/s00248-003-2022-7.

45. Kumar PKR, Schugerl K. 1990. Immobilization of genetically engineered cells - a new strategy for higher stability. J Biotechnol 14:255-272. https:// doi.org/10.1016/0168-1656(90)90111-N.

46. Shinmyo A, Kimura H, Okada H. 1982. Physiology of alpha-amylase production by immobilized Bacillus amyloliquefaciens. Eur J Appl Microbiol Biotechnol 14:7-12. https://doi.org/10.1007/BF00507996.

47. de Taxis Du Poët P, Arcand Y, Bernier R, Barbotin JN, Thomas D. 1987. Plasmid stability in immobilized and free recombinant Escherichia coli Jm105(Pkk223-200)_importance of oxygen diffusion, growth rate, and plasmid copy number. Appl Environ Microbiol 53:1548-1555. https://doi .org/10.1128/AEM.53.7.1548-1555.1987.

48. Stewart PS, Robertson CR. 1988. Product inhibition of immobilized Escherichia coli arising from mass transfer limitation. Appl Environ Microbiol 54:2464-2471. https://doi.org/10.1128/AEM.54.10.2464-2471.1988.

49. Bron PA, Grangette C, Mercenier A, de Vos WM, Kleerebezem M. 2004. Identification of Lactobacillus plantarum genes that are induced in the gastrointestinal tract of mice. J Bacteriol 186:5721-5729. https://doi.org/ 10.1128/JB.186.17.5721-5729.2004.

50. Marco ML, Peters TH, Bongers RS, Molenaar D, van Hemert S, Sonnenburg JL, Gordon J, Kleerebezem M. 2009. Lifestyle of Lactobacillus plantarum in the mouse caecum. Environ Microbiol 11:2747-2757. https://doi.org/10 .1111/j.1462-2920.2009.02001.x.

51. Remus DM, van Kranenburg R, van Swam II, Taverne N, Bongers RS, Wels M, Wells JM, Bron PA, Kleerebezem M. 2012. Impact of 4 Lactobacillus plantarum capsular polysaccharide clusters on surface glycan composition and host cell signaling. Microb Cell Fact 11:149. https://doi.org/10 .1186/1475-2859-11-149.

52. Michel C, Kravtchenko TP, David A, Gueneau S, Kozlowski F, Cherbut C. 1998. In vitro prebiotic effects of Acacia gums onto the human intestinal microbiota depends on both botanical origin and environmental $\mathrm{pH}$. Anaerobe 4:257-266. https://doi.org/10.1006/anae.1998.0178. 
53. Tanner SA, Chassard C, Zihler Berner A, Lacroix C. 2014. Synergistic effects of Bifidobacterium thermophilum RBL67 and selected prebiotics on inhibition of Salmonella colonization in the swine proximal colon PolyFermS model. Gut Pathog 6:44. https://doi.org/10.1186/s13099-014-0044-y.

54. Poeker SA, Geirnaert A, Berchtold L, Greppi A, Krych L, Steinert RE, de Wouters T, Lacroix C. 2018. Understanding the prebiotic potential of different dietary fibers using an in vitro continuous adult fermentation model (PolyFermS). Sci Rep 8:4318. https://doi.org/10.1038/s41598-018 -22438-y.

55. Bircher L, Geirnaert A, Hammes F, Lacroix C, Schwab C. 2018. Effect of cryopreservation and lyophilization on viability and growth of strict anaerobic human gut microbes. Microb Biotechnol 11:721-733. https://doi.org/ 10.1111/1751-7915.13265.

56. Topping DL, Clifton PM. 2001. Short-chain fatty acids and human colonic function: roles of resistant starch and nonstarch polysaccharides. Physiol Rev 81:1031-1064. https://doi.org/10.1152/physrev.2001.81.3.1031.

57. Martin M. 2011. Cutadapt removes adapter sequences from high-throughput sequencing reads. EMBnet J 17:10-12. https://doi.org/10.14806/ej.17.1 200 .

58. Callahan BJ, McMurdie PJ, Rosen MJ, Han AW, Johnson AJA, Holmes SP. 2016. DADA2: high-resolution sample inference from Illumina amplicon data. Nat Methods 13:581-583. https://doi.org/10.1038/nmeth.3869.
59. Quast C, Pruesse E, Yilmaz P, Gerken J, Schweer T, Yarza P, Peplies J, Glockner FO. 2013. The SILVA ribosomal RNA gene database project: improved data processing and web-based tools. Nucleic Acids Res 41:D590-D596. https:// doi.org/10.1093/nar/gks1219.

60. Filannino P, De Angelis M, Di Cagno R, Gozzi G, Riciputi Y, Gobbetti M. 2018. How Lactobacillus plantarum shapes its transcriptome in response to contrasting habitats. Environ Microbiol 20:3700-3716. https://doi.org/ 10.1111/1462-2920.14372.

61. Jans C, Gerber A, Bugnard J, Njage PMK, Lacroix C, Meile L. 2012. Novel Streptococcus infantarius subsp infantarius variants harboring lactose metabolism genes homologous to Streptococcus thermophilus. Food Microbiol 31:33-42. https://doi.org/10.1016/j.fm.2012.02.001.

62. Stevens MJA, Zurfluh K, Stephan R. 2018. Complete and assembled genome sequences of Pantoea calida DSM 22759(T) and Pantoea gaviniae DSM 22758(T). Genome Announc 6:e00157-18. https://doi.org/10.1128/ genomeA.00157-18.

63. Treangen TJ, Ondov BD, Koren S, Phillippy AM. 2014. The Harvest suite for rapid core-genome alignment and visualization of thousands of intraspecific microbial genomes. Genome Biol 15:524. https://doi.org/ 10.1186/s13059-014-0524-x.

64. Edgar RC. 2004. MUSCLE: multiple sequence alignment with high accuracy and high throughput. Nucleic Acids Res 32:1792-1797. https://doi .org/10.1093/nar/gkh340. 